\title{
Smoking Related Attention Alteration in Chronic Obstructive Pulmonary Disease-Smoking Comorbidity
}

\section{Feiyan Zeng}

The First Affiliated Hospital of USTC: Anhui Provincial Hospital

\section{Wei Hong}

University of Science and Technology of China

\section{Rujing Zha}

University of Science and Technology of China

\section{Ying Li}

University of Science and Technology of China

\section{Ying Liu}

The First Affiliated Hospital of USTC: Anhui Provincial Hospital

\section{Hao Liu}

University of Science and Technology of China

\section{Mengqiu Liu}

The First Affiliated Hospital of USTC: Anhui Provincial Hospital

\section{Mei Liu}

The First Affiliated Hospital of USTC: Anhui Provincial Hospital

\section{Fei Xu}

The First Affiliated Hospital of USTC: Anhui Provincial Hospital

\section{Daiju Hu}

The First Affiliated Hospital of USTC: Anhui Provincial Hospital

\section{Hongwen Song}

University of Science and Technology of China

\section{Yongqiang Yu}

The First Affiliated Hospital of USTC: Anhui Provincial Hospital

Xiaochu Zhang ( $\nabla$ zxcustc@ustc.edu.cn)

University of Science and Technology of China https://orcid.org/0000-0002-7541-0130

\section{Research Article}

Keywords: Chronic obstructive pulmonary disease, smoking, comorbidity, attention ability, functional magnetic resonance imaging 
Posted Date: June 14th, 2021

DOl: https://doi.org/10.21203/rs.3.rs-594226/v1

License: (c) (i) This work is licensed under a Creative Commons Attribution 4.0 International License. Read Full License 
Smoking related attention alteration in Chronic Obstructive Pulmonary Disease-Smoking Comorbidity

Feiyan Zeng', Wei Hong', Rujing Zha' ${ }^{2 *}$ Ying $\mathrm{Li}^{2}$, Ying Liu', Hao Liu ${ }^{3}$, Mengqiu

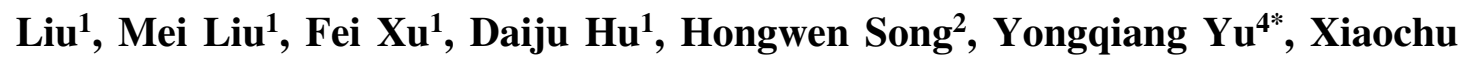

$9{ }^{1}$ The First Affiliated Hospital of USTC, Division of Life Sciences and Medicine, 10 University of Science and Technology of China, Hefei, Anhui, 230001, China.

$11{ }^{2}$ Key Laboratory of Brain Function and Disease, Chinese Academy of Sciences, School 12 of Life Sciences, Division of Life Sciences and Medicine, University of Science and 13 Technology of China, Hefei, Anhui, 230027, China.

$14{ }^{3}$ School of Earth and Space Science, University of Science and Technology of China, 15 Hefei, Anhui, 230027, China.

$16{ }^{4}$ Department of Radiology, The First Affiliated Hospital of Anhui Medical University, 17 Hefei, Anhui, 230022, China.

18 CORRESPONDENCE TO

19 Rujing Zha, E-mail: zharj@ustc.edu.cn (R. Zha)

20 Yongqiang Yu, E-mail: cjr.yuyongqiang@vip.163.com (Y. Yu).

21 Xiaochu Zhang, E-mail: zxcustc@ustc.edu.cn (X. Zhang), Tel: +86-551-63607295 
Co-first author

24 Feiyan Zeng, Wei Hong and Rujing Zha are co-first authors

25 Abstract word count: 216 words

26 Word count: 2996 words 


\section{Abstract}

Chronic obstructive pulmonary disease is a respiratory disease that causes a wide range of cognitive impairments. Although chronic obstructive pulmonary disease-smoking comorbidity is common, the relationship between smoking and cognitive function in chronic obstructive pulmonary disease-smoking comorbidity remains unclear. In this study, we recruited 85 participants, including 42 patients with Chronic obstructive pulmonary disease(20 smokers and 22 non-smokers) and 43 healthy participants(22 smokers and 21 non-smokers). And we used the Montreal Cognitive Assessment scale and resting-state functional magnetic resonance imaging to explore effects of smoking on attention in patients with chronic obstructive pulmonary disease. Behavioral analysis revealed that smokers had a shorter course of chronic obstructive pulmonary disease and showed a worse attention performance in chronic obstructive pulmonary diseasesmoking comorbidity compared to non-smokers. Resting-state functional magnetic resonance imaging analysis revealed that smokers showed lower regional homogeneity value of the fusiform gyrus in chronic obstructive pulmonary disease-smoking comorbidity than non-smokers. Importantly, the regional homogeneity of the fusiform gyrus was positively associated with attention and mediates the effect of smoking on attention in chronic obstructive pulmonary disease. In summary, our study provides behavioral and neurobiological evidence supporting the positive effect of smoking on attention in chronic obstructive pulmonary disease. This may be helpful for understanding and treating chronic obstructive pulmonary disease and even other diseases comorbid with smoking. 
Key words: Chronic obstructive pulmonary disease, smoking, comorbidity, attention ability, functional magnetic resonance imaging

\section{Introduction}

Chronic obstructive pulmonary disease(COPD) is a chronic respiratory disease and one of the leading causes of morbidity and mortality worldwide(Spilling et al., 2019). It is characterized by persistent respiratory symptoms and limited airflow(Borson, 2008; Martinez, Richardson, Han, \& Cigolle, 2014; Roncero, Campuzano, Quintano, Molina, $\&$ Miravitlles, 2016) and is an independent risk factor for cognitive impairment(Orua, 2013; Roberto, Luca, Silvia, Fernanda, \& Paola, 2014). Poor cognitive function in patients with COPD may result in a greater risk of hospitalization, longer hospital stays, and worsening health status, which may lead to an increased mortality rate(Dodd, Charlton, Van, \& Jones, 2013; Fix, Daughton, Kass, \& Bell, 2009). Therefore, it is of great clinical significance to explore the mechanism of impaired cognitive function in COPD patients.

Smoking has been recognized as the leading cause of chronic obstructive pulmonary disease, as the long-term stimulation of cigarette smoke eventually leads to COPD(Eltom, Stevenson, \& Birrell, 2013; Forey, Thornton, \& Lee, 2011). Studies have shown that approximately $80 \%$ $90 \%$ of COPD patients have a history of smoking, and half of smokers will eventually develop COPD(Lundbäck, Lindberg, \& Lindström, 2003). Despite the prevalence of the comorbidity between COPD and smoking, the role 
71 of smoking in cognition and brain damage in COPD-smoking comorbidity is largely 72 unclear.

A line of studies has demonstrated the existence of cognitive impairments in COPD.

74 A recent review found that COPD patients were commonly exhibit deficits in attention, 75 memory, executive function, psychomotor function, and language(Olaithe, Bucks, 76 Hillman, \& Eastwood, 2018). Specifically, compared former smokers with and without 77 COPD and found that smokers with COPD had impairment in attention, characterized by slow processing, inattention, and impulsivity(Croghan et al., 2019). And patients with COPD had insufficient attentional resources for successfully dealing with dual tasks, which led to greater gait variability(Nelly et al., 2018). Another line of studies revealed abnormal brain structure and function in COPD patients. For example, the grey matter volume in the bilateral fusiform gyrus, bilateral calcarine, right superior temporal gyrus, right middle temporal gyrus, left precuneus and right inferior parietal lobule were significantly reduced in COPD patients compared with normal controls, and their forced vital capacity(FVC\%) was closely related to the volume of cortical grey matter(Wang et al., 2017). Functional connectivity analysis found that the connectivity density of the right lingual gyrus(LG), bilateral supplementary motor area(SMA) and right paracentral lobules(PCL) was significantly reduced in COPD patients compared with normal controls( $\mathrm{Li}, \mathrm{Xin}, \mathrm{Yu}, \mathrm{Yu}, \&$ Peng, 2019). Further seed-based functional connection analysis revealed that COPD patients had decreased functional connections in the left anterior cerebellar lobe, left fusiform gyrus, right insula lobe, right inferior frontal lobe, left putamen and other regions. 

has shown that smoking itself causes attention disability. Compared with non-smokers, smokers had an increased risk of cognitive impairment(Domino, 2008). Smoking also leads to chronic attention maintenance deficits(Konishi et al., 1999). Another line of studies revealed abnormal brain structure and function in smokers. Using smokingrelated images can increase the activation of brain regions related to visual spatial attention, such as the bilateral parietal lobe and fusiform gyrus(Due, Huettel, Hall, \& Rubin, 2002). Long-term smoking can cause damage to the prefrontal cortex, which

101 leads to long-term attention maintenance regulation deficits(Konishi et al., 1999).

102 Smokers had significantly smaller grey matter volume and lower grey matter density in

103 the anterior cingulate, prefrontal cortex, orbitofrontal cortex, occipital lobe, temporal

104 lobe and parahippocampal gyrus. The amount of tobacco smoke exposure was 105 negatively correlated with the volume of the frontal lobe, temporal lobe, and cerebellum.

106 Structural damage in the cortex and subcortical regions of smokers was associated with 107 deficits in the brain networks of attention and working memory(Jürgen et al., 2006).

108 Although smoking leads to extensive damage to brain structures and function, its 109 neuroprotective effect has been found in numerous molecular and cellular biological 110 studies on nicotine in cigarette smoke(Ward, Lallemand, Witte, \& Dexter, 2008).

111 Nicotine also has a protective effect on dopaminergic neurons. In addition to potential 112 neuroprotective behaviors, from learning and memory enhancement to addiction and 113 neuroprotection, nicotine also has antidepressant properties and the ability to improve 
114 cognitive activities such as attention(DAJASBAILADOR, 2004) and cognitive

115 control(Evans, To, \& Ashare, 2018).

116 On the one hand, studies on the long-term effect of smoking have shown smoking

117 impaired attention ability(Due et al., 2002), we expect that smoking aggravates

118 attention and neural impairment in COPD patients with a smoking comorbidity. On the

119 other hand, research on the acute effect of smoking proved that smoking could improve

120 attention(Ward et al., 2008), we expect that smoking may related to attention and neural

121 impairment in COPD patients with a smoking comorbidity.

122 In the present study, first explored the effect of smoking on attention in COPD

123 patients with a smoking comorbidity and located regions of interest(ROIs) whose

124 regional homogeneity $(\mathrm{ReHo})$ showed alterations in this population. Finally, tested the

125 relationship between these brain regions and smoking and attention ability.

\section{Materials and Methods}

\section{Study Design}

\subsection{Procedure}

Each subject underwent a pulmonary function test, resting-state functional

132 magnetic resonance imaging(fMRI) scan, Montreal Cognitive Assessment(MoCA)

133 Scale(Dalrymple-Alford et al., 2010), demographic survey and smoking behavior

134 assessment only for smokers. Details see supplementary materials. 


\subsection{Participants}

We recruited 85 participants, including 20 smoking COPD patients(COPDSmoking group), 22 non-smoking COPD patients(COPD-Nonsmoking group), 22 smoking healthy subjects(NonCOPD-Smoking group) and 21 non-smoking healthy subjects(NonCOPD-Nonsmoking group), shown in TableS1 and TableS2. Inclusion and exclusion criteria see supplementary materials.

\subsection{Resting-state fMRI analysis}

\subsubsection{Preprocessing}

Considering the instability of the instrument and the subjects at the beginning of the scanning, the first $10 \mathrm{TR}$ of the scanning were excluded. Then slice time correction was performed at the remaining time points to reduce the heterogeneity of the data at different levels due to different acquisition times. Spatial correction was performed to correct for the effect of the head motion, any TR with head motion $>2.5 \mathrm{~mm}$ of translation or $2.0^{\circ}$ of rotation during the scan were excluded. The resting state functional image was registered to the structure image, and the space is normalized to the MNI standard space. For nuisance signal correction, the following nuisance parameters were included as regressors within the general linear model; 6 motion parameters and their first derivatives, white matter(WM), cerebrospinal fluid(CSF) and a linear trend term. Gaussian kernel(FWHM=6mm) was used for spatial smoothing to reduce the influence of spatial noise on scanning and improve the signal-to-noise ratio. 
157 Linear drift removal and filtering were performed. $0.01-0.1 \mathrm{~Hz}$ frequency band was

158 adopted to filter out physiological noise such as breathing and heartbeat.

159

160

161

162

163

164

165

166

167

168

169

170

171

172

173

174

175

\subsubsection{Functional connectivity}

After processing, we obtained the time courses of the scans of the whole brain. Then, the whole brain was divided into 90 regions according to the Anatomical Automatic Labelling(AAL) parcellation atlas(Tzourio-Mazoyer et al., 2002). Each region was calculated as an average time series and then correlated with the time series of all other regions to obtain a $90 * 90$ correlation matrix.

\subsubsection{ReHo}

ReHo was calculated in each region by REST software(http://www.restfmri.net/). Each voxel and its adjacent voxels were selected to calculate the ReHo in the same time series, which was then assigned to the initial voxel. The ReHo values were standardized and spatial smoothed. Statistical Parametric Mapping 8(SPM8)(Ashburner \& Friston, 2005) was used to perform two-sample T-tests with whole-brain and threshold using family wise error correction(FWER) at $\mathrm{p}<0.05$.

\subsubsection{Node betweenness centrality}

According to graph theory, the node betweenness centrality of each brain region was calculated based on functional connectivity. Node betweenness centrality is the fraction of all shortest paths in a network that contain a given node. In this study, the node betweenness centrality was calculated by the tools implemented in the Brain Connectivity Toolbox(version 2015-01-25)(Rubinov \& Sporns, 2010). 


\subsection{Statistical analysis}

Two-way ANOVA was used to analyze the effects of smoking and COPD on

181 cognitive function. Independent samples $\mathrm{T}$ tests were used to compare age, years of

182 education, smoking index, smoking history and pulmonary function(FEV1\%, FVC\%

183 and FEV1/FVC) between the COPD group and the NonCOPD group, as well as the

184 cognitive functions, smoking index, smoking history, ReHo and node betweenness

185 centrality of brain regions between the COPD-Smoking group and the COPD-

186 Nonsmoking group. The Cohen's d values were calculated via G*Power 3.1

187 software(Faul, Erdfelder, Lang, \& Buchner, 2007). The $\chi 2$ test was used to compare

188 gender between the COPD group and the NonCOPD group. Pearson's correlation was

189 used to analyze the relationship between smoking index/smoking history and the ReHo.

190 Kendall's tau-b correlation analysis was used to analyze the relationship between the

191 ReHo and MoCA cognitive scores, as well as between smoking index and MoCA

192 cognitive scores, since the MoCA scores are rank variables. Bootstrap mediation

193 analysis(Wen, Marsh, \& Hau, 2010) was used to analyze the medicating effect of ReHo

194 on smoking index and attention. Finally, considering the imbalance of sex ratio

195 between smokers and non-smokers, we performed covariance analysis using gender as

196 a covariable in the analysis of attention. 


\section{Results}

\subsection{Smokers had a shorter course of COPD in COPD-smoking comorbidity}

There were no significant differences in gender, age and years of education between the COPD group and the NonCOPD group(TableS1). There was no significant

204 differences in smoking $\operatorname{index}\left(t_{40}=0.44, p=0.665, d=0.136\right)$ and smoking history( $t_{40}=0.88, p=0.383, d=0.272$ ) between the COPD-Smoking and NonCOPD-Smoking groups .

First, we analyzed the impact of smoking on the course of COPD and found that

208 the course of COPD in the COPD-Nonsmoking group was significantly higher than that 209 in the COPD-Smoking $\operatorname{group}\left(t_{40}=3.51, p=0.001, d=1.086\right)$. Smoking occurred 210 earlier than COPD in the COPD-Smoking group $\left(t_{19}=17.81, p<0.001, d=3.982\right)$.

211 However, there were no significant differences in FEV1\%( $t_{40}=-0.74, p=$ $212 \quad 0.463, d=-0.229 \quad), \quad \operatorname{FVC} \%\left(\quad t_{40}=0.81, p=0.424, d=0.250 \quad\right) \quad$ and

213 FEV1/FVC $\left(t_{40}=-1.43, p=0.163, d=-0.442\right)$ between the COPD-Smoking 214 group and COPD-Nonsmoking group, which suggested that COPD causes a consistent 215 lung condition in these two group. 

compared to non-smokers

To understand more about the effect of smoking on attention in COPD patients, two-way ANOVA of the MoCA score was performed and revealed a significant interaction effect $($ COPD $\times$ smoking $)\left(F_{(1,81)}=5.91, p=0.017\right.$, partial $\eta^{2}=$ 0.068)on attention ability and a significant main effect of $\operatorname{COPD}\left(F_{(1,81)}=37.94, p<\right.$ 0.001, partial $\left.\eta^{2}=0.319\right)$ and of smoking on the scores of attention $\operatorname{ability}\left(F_{(1,81)}=4.90, p=0.030\right.$, partial $\left.\eta^{2}=0.057\right)$, which suggest that smokers showed less impairment of attention disability(Figure1). In particular, the score of attention ability $\left(t_{40}=2.53, p=0.016, d=0.781\right.$ ) was higher in the COPDSmoking group than in the COPD-Nonsmoking group. In addition, the analysis of covariance including sex still showed significant interaction effects $(\mathrm{COPD} \times$ smoking $)\left(F_{(1,80)}=5.82, p=0.018\right.$, partial $\left.\eta^{2}=0.068\right)$.

3.3 Smoker showed lower ReHo value in COPD-smoking comorbidity compared to non-smokers

To explore the neural mechanisms of cognitive impairment in COPD, we 235 performed whole-brain $\mathrm{T}$ test analysis. The results showed that the ReHo of the six 236 clusters in the COPD group, which were located in the bilateral fusiform gyrus, left 237 inferior temporal gyrus, left anterior cerebellar lobe, and pons, were lower than those 238 in the NonCOPD group after FWER correction(Table1 and Figure2). 
In particular, the ReHo of the left fusiform gyrus $\left(t_{40}=2.09, p=0.043, d=\right.$

0.646 ), right fusiform gyrus( $\left.t_{40}=2.96, p=0.005, d=0.915\right)$, left anterior

241 cerebellum $\left(t_{40}=2.29, p=0.027, d=0.708\right)$ and $\operatorname{pons}\left(t_{40}=2.56, p=0.014, d=\right.$

0.791) were all higher in the COPD-Nonsmoking group than in the COPD-Smoking group(FigureS2).

3.4 ReHo and functional connectivity of the left fusiform gyrus correlate with

\section{attention ability}

Kendall's tau-b correlation analysis was used to analyze the relationship between the ReHo and MoCA cognitive scores, as well as between smoking index and MoCA cognitive scores, since the MoCA scores are rank variables. The analysis showed that the ReHo of the left fusiform gyrus $\left(r_{42}=0.333, p=0.005\right)$, right fusiform $\operatorname{gyrus}\left(r_{42}=0.259, p=0.030\right)$ and left inferior temporal gyrus $\left(r_{42}=0.316, p=\right.$ 0.008) were associated with attention ability in the COPD groups.

Two-way ANOVA of node betweenness centrality showed a main effect of 254 smoking in the right fusiform $\operatorname{gyrus}\left(F_{(1,81)}=4.40, p=0.039\right.$, partial $\left.\eta^{2}=0.052\right)$ 255 and a main effect of COPD in the left fusiform gyrus $\left(F_{(1,81)}=25.39, p<\right.$ 2560.001, partial $\left.\left.\eta^{2}=0.239\right)\right)$ and right fusiform $\operatorname{gyrus}\left(F_{(1,81)}=14.79, p<\right.$ 2570.001 , partial $\left.\eta^{2}=0.154\right)$. However, there was no significant interaction effect 258 (COPD $\times$ smoking $)($ FigureS3). 
261 functional connectivity between them and all other brain regions. Kendall's tau-b 262 correlation analysis found that the functional connectivity between left fusiform gyrus 263 and left pallidum was positively correlated with attention ability $\left(r_{42}=0.253, p=\right.$ $2640.033)$ and total $\operatorname{MoCA} \operatorname{score}\left(r_{42}=0.338, p=0.002\right)$ in the COPD groups.

3.5 Mediating analysis: ReHo of the left fusiform gyrus mediated the influence of smoking on attention ability

To understand more about the mechanism of the impact of smoking on cognitive function in COPD patients, we analyzed the relationships between smoking index and cognitive function and the ReHo. First, Kendall's tau-b correlation analysis found that

271 the smoking index was positively related to attention ability in COPD patients $\left(r_{42}=\right.$ $0.336, p=0.030$ ), including the COPD-Smoking group and COPD-Nonsmoking 273 group. Pearson's correlation was used to analyze the relationship between smoking 274 index/smoking history and the ReHo, since these are all continuous variables, and found 275 that the smoking index was negatively related to the ReHo of the left fusiform gyrus in 276 COPD patients $\left(r_{39}=-0.345, p=0.025\right)$. Mediation analysis revealed that the ReHo 277 of the left fusiform gyrus completely mediated the effect of the smoking index on 278 attention ability in COPD patients ( LLCI ULCI: $-0.0002 \sim 0.0011$, 279 BootLLCI BootULCI : $0.0001 \sim 0.0008$ ) but not in NonCOPD group(Figure3). 


\section{Discussion}

COPD is often comorbid with smoking; however, the way in which smoking modulates behavior and its neural basis in COPD-smoking comorbidity remains unclear. At the behavioral level, we found that smokers showed less attention impairment in COPD-smoking comorbidity. At the neural level, we found that the ReHo of the left 287 fusiform gyrus completely mediated the effect of smoking on attention ability. Totally, 288 the funding of this study suggested that smokers showed less impairment of attention 289 ability in COPD-smoking comorbidity, which related to the function of left fusiform gyrus.

Not only is nicotine an important component in cigarettes that causes smoking addiction but it also has a protective effect on dopaminergic neurons. Neuronal nicotinic acetylcholine receptors in the brain are more often associated with neuromodulation

294 than mediation of synaptic transmission. The complex $\mathrm{Ca}^{2+}$ response generated by the 295 activation of the nicotinic acetylcholine receptor can transmit information beyond the 296 initial domain and promote the activation of many intracellular signaling pathways. 297 These mechanisms form the basis for the diversity of nicotinic neuron activity in the 298 brain, from the enhancement of learning and memory to addiction and 299 neuroprotection(DAJASBAILADOR, 2004). Thus, the protective mechanism of 300 nicotine may be responsible for the less cognitive impairment in COPD-smoking 301 comorbidity. Moreover, smoking occurred earlier than COPD in the COPD-Smoking 
group. Presumably, smoking may preempt a protective mechanism that buffers cognitive impairment in COPD-smoking comorbidity.

We found that the ReHo of the left fusiform gyrus mediates the influence of smoking on attention ability, which suggests that it may be the central brain region of the acute effect of smoking. The fusiform gyrus is an integral part of the ventral occipito-temporal conjoint brain region. Previous studies have shown that this region is widely involved in cognitive processes such as face recognition, object position and vocabulary and is associated with visual spatial attention(Han et al., 2012). Thus, the

310 fusiform gyrus is likely a central factor in the effect of smoking on attention ability in 311 COPD-smoking comorbidity. In addition, whole-brain analysis also found differences 312 in left inferior gyrus, left anterior cerebellum and pons between COPD and non-COPD 313 groups. Previous studies have shown that inferior gyrus(IFG) maybe related with the 314 phonological information storage, behavior chosen or attention modulation(Yang, Xiao, 315 Liu, Weng, \& Zhang, 2006). And the neural network involved in the age-related 316 decrements in memory and attention included IFG and the cerebellum(Iidaka et al., 317 1999), which means these brain regions may be the key factor in cognitive impairment 318 for COPD patients.

319 We were also concerned about other cognitive functions in the MoCA, including 320 visuospatial execution ability, naming ability, language ability, abstraction ability and 321 delayed recall ability. We found that these functions were all impaired in COPD 322 patients(Table S3). This finding is consistent with previous studies(Antonelli-Incalzi et 323 al., 2008; Villeneuve, Pépin, Rahayel, Bertrand, \& Gagnon, 2012; Zhang H, Wang X, 
\& Lin J, 2012). Specifically, only visuospatial execution ability and abstraction ability were significantly worse in COPD-Nonsmoking than in COPD-Smoking(FigureS1 and TableS6), but no mediation effect was found. In addition, researchers have found that smoking also harms cognitive functions independently, such as memory(Liu et al., 2013) and execution ability(Crean, Crane, \& Mason, 2011). In this study, we also found that delayed recall ability was worse in the NonCOPD-Smoking group than in the

330 NonCOPD-Nonsmoking group(FigureS1). Overall, the independent effects of smoking and COPD on other cognitive functions are consistent with previous studies. The role of smoking in the impairment of these cognitive functions in COPD patients may be similar to that in the impairment of attention ability, but the neural mechanisms remain unclear and deserve further study.

Although we aimed to ensure a high-quality study, there were still some limitations.

336 First, there is imbalance of sex ratio between smokers and non-smokers, which is due

337 to very low prevalence $(2.7 \%)$ of female Chinese smokers and the possible influence of 338 the menstrual cycle phase on smoking cue reactivity and cigarette craving(Franklin et 339 al., 2015). Tring to eliminate the influence of sex on the results of this study, we 340 performed covariance analysis on the main results using sex as a covariable, and the 341 results was consistent(see Results). Second, the subjects in this study were older and 342 generally less educated, which may have led to an over evaluation of the degree of 343 cognitive decline. Third, the sample size of this study was too small to stratify disease 344 according to severity. It is hoped that in future work, more female smokers, more 
346 be conducted according to the severity of the disease or other factors.

\section{Conclusions}

This study revealed the effect of smoking on attention ability in COPD-smoking

351 comorbidity. Smokers showed less attention impairment in COPD-smoking 352 comorbidity, which mediated by the ReHo of the fusiform gyrus. The results support 353 the acute effect of smoking on behaviors in COPD-smoking comorbidity. While 354 previous studies have tended to exclude smoking from their focus on COPD, the 355 findings in this paper are the first to reveal the positive aspect of smoking in COPD, 356 suggesting an important role of smoking in COPD-smoking comorbidity and 357 potentially in other diseases.

\section{Acknowledgments}

360 A portion of the numerical calculations in this study were performed with the 361 supercomputing system at the Supercomputing Centre of USTC. We also thank the 362 Bioinformatics Center of the University of Science and Technology of China, School 363 of Life Science, for providing supercomputing resources for this project. 


\section{Declarations}

\section{Funding}

This work was supported by grants from The National Key Basic Research

Program (2018YFC0831101), The National Natural Science Foundation of China

Social Science Research, Ministry of Education of China (19JZD010), CAS-VPST Silk

Road Science Fund 2021 (GLHZ202128), Collaborative Innovation Program of Hefei

Science Center, CAS (2020HSC-CIP001).

373 corresponding authors with reasonable request.

\section{Competing interests}

\section{Ethics approval} number is $2020-\mathrm{N}(\mathrm{H})-186$.

\section{Consent to Participate}

\section{Consent to Publish}

We consent to publication of this paper.

\section{Availability of data and materials}

All authors claim that there are no conflicts of interest.

This study was approved by the Institutional Review Boards of University of Science and Technology of China and Anhui Provincial Hospital. And the IRB approval

Informed consent was obtained from all participants in the study.

Additional material and all other data for this study can be available from the 


\section{Code Availability}

Code will be provided to qualified researchers upon reasonable request.

\section{Author contributions}

Feiyan Zeng, Wei Hong, Rujing Zha, Yongqiang Yu and Xiaochu Zhang contributed to the study design. All authors contributed to the acquisition, analysis, or interpretation of the data. Feiyan Zeng, Wei Hong, Rujing Zha and Xiaochu Zhang contributed to the critical revision of the manuscript for important intellectual content.

Feiyan Zeng, Wei Hong, Rujing Zha, Ying Liu, Hao Liu contributed to the statistical analysis.

\section{Additional information}

Additional supporting information may be found in the Supporting Information section at the end of this article.

\section{REFERENCES}

Antonelli-Incalzi, R., Corsonello, A., Trojano, L., Acanfora, D., Spada, A., Izzo, dependence in hypoxemic COPD. J Clin Exp Neuropsychol, 30(2), 141-150. Ashburner, J., \& Friston, K. J. (2005). Unified segmentation. NeuroImage, 26, 839851.

Borson, S. (2008). Modeling the impact of COPD on the brain. International Journal of $C O P D, 3(3)$.

Crean, R., Crane, N., \& Mason, B. (2011). An Evidence-Based Review of Acute and Long-Term Effects of Cannabis Use on Executive Cognitive Functions. JOURNAL OF ADDICTION MEDICINE, 5(1), 1-8.

Croghan, A., Brunette, A., Holm, K. E., Kozora, E., Moser, D. J., Wamboldt, F. S., . . Hoth, K. F. (2019). Reduced Attention in Former Smokers with and without COPD. Int J Behav Med, 26(6), 600-607. Retrieved from https://www. ncbi.nlm. nih. gov/pubmed/31732904 
DAJASBAILADOR, F. (2004). Nicotinic acetylcholine receptors and the regulation of neuronal signalling. Trends in Pharmacological ences, 25(6), 317-324.

Dalrymple-Alford, J. C., Macaski11, M. R., Nakas, C. T., Livingston, L., Graham, C., Crucian, G. P., . . . Wells, S. (2010). The MoCA: We11-suited screen for cognitive impairment in Parkinson disease. Neurology.

Dodd, J. W., Charlton, R. A., Van, d. B., Martin D., \& Jones, P. W. (2013). Cognitive Dysfunction in Patients Hospitalized With Acute Exacerbation of COPD. Chest, 144(1), 119-127.

Domino, E. F. (2008). Tobacco smoking and MRI/MRS brain abnormalities compared to nonsmokers. Progress in Neuropsychopharmacology Biological Psychiatry, $32(8), \quad 1778-1781$.

Due, D. L., Huettel, S. A., Hall, W. G., \& Rubin, D. C. (2002). Activation in mesolimbic and visuospatial neural circuits elicited by smoking cues: evidence from functional magnetic resonance imaging. Am J Psychiatry, 159(6), 954-960. doi:10. 1176/appi. ajp. 159.6. 954

E1tom, S., Stevenson, C., \& Birre11, M. A. (2013). Cigarette Smoke Exposure as a Model of Inflammation Associated with COPD. Curr Protoc Pharmacol, Chapter $5(1)$.

Evans, D. E., To, C. N., \& Ashare, R. L. (2018). The Role of Cognitive Control in the Self-Regulation and Reinforcement of Smoking Behavior. Nicotine \& Tobacco Research, 21(6), 747-754. doi:10. 1093/ntr/nty029 \%J Nicotine \& Tobacco Research

Faul, F., Erdfelder, E., Lang, A. -G. , \& Buchner, A. (2007). G* Power 3: A flexible statistical power analysis program for the social, behavioral, and biomedical sciences. Behavior research methods, 39(2), 175-191.

Fix, A. J., Daughton, D., Kass, I., \& Be11, C. W. (2009). Cognitive Functioning and Survival Among Patients with Chronic Obstructive Pulmonary Disease. International Journal of Neuroscience.

Forey, B. A., Thornton, A. J., \& Lee, P. N. (2011). Systematic review with metaanalysis of the epidemiological evidence relating smoking to COPD, chronic bronchitis and emphysema. BMC Pulm Med, 11(36).

Franklin, T. R., Jagannathan, K., Wetheri11, R. R., Johnson, B., Kelly, S., Langguth, J., . . . Childress, A. R. (2015). Influence of Menstrual Cycle Phase on Neural and Craving Responses to Appetitive Smoking Cues in Naturally Cycling Females. Nicotine \& Tobacco Research, 17(4), 390-397. doi:10. 1093/ntr/ntu183 \%J Nicotine \& Tobacco Research

Han, Y., Lui, S., Kuang, W., Lang, Q., Zou, L., \& Jia, J. (2012). Anatomical and Functional Deficits in Patients with Amnestic Mild Cognitive Impairment. PLoS One.

Iidaka, T., Anderson, N., Kapur, S., Cabeza, R., Brain, F. C. J., \& Cognition. (1999). Age-related differences in brain activation during encoding and retrieval under divided attention: A positron emission tomography (PET) study. 39(1), 53-55.

Jürgen, Gallinat, Eva, Meisenzahl, Leslie, K., . . . Jeffrey. (2006). Smoking and 
structural brain deficits: a volumetric MR investigation. European Journal of Neuroscience.

Konishi, S., Nakajima, K., Uchida, I., Kikyo, H., Kameyama, M. , \& Miyashita, Y. (1999). Common inhibitory mechanism in human inferior prefrontal cortex revealed by event-related functional MRI. 122.

Li, H., Xin, H., Yu, J., Yu, H., \& Peng, D. (2019). Abnormal intrinsic functional hubs and connectivity in stable patients with COPD: a resting-state MRI study. Brain Imaging Behavior(1).

Liu, J. T., Lee, I. H., Wang, C. H., Chen, K. C., Lee, C. I., \& Yang, Y. K. (2013). Cigarette smoking might impair memory and sleep quality. Journal of the Formosan Medical Association, 112(5), 287-290.

Lundbäck, B., Lindberg, A., \& Lindström, M. (2003). Not 15 but 50\% of smokers develop COPD ? Report from the Obstructive Lung Disease in Northern SwedenStudies. Respir Med, 97(2), 115-122.

Martinez, C. H., Richardson, C. R., Han, M. L. K., \& Cigolle, C. T. (2014). Chronic Obstructive Pulmonary Disease, Cognitive Impairment, and Development of Disability: The Health and Retirement Study. Annals of the American Thoracic Society, 11(9), 1362-1370.

Nelly, H. , Fran?0is, A., Mathieu, G., Corentin, D., Emilie, T., Nicolas, 0., \& Alain, V. J. C. J. o. C. 0. P. D. (2018). Impact of Chronic Obstructive Pulmonary Disease on Cognitive and Motor Performances in Dual-Task Walking. $1-6$.

Olaithe, M., Bucks, R. S., Hillman, D. R., \& Eastwood, P. R. (2018). Cognitive deficits in obstructive sleep apnea: Insights from a meta-review and comparison with deficits observed in COPD, insomnia, and sleep deprivation. Sleep Med Rev, 38, 39-49. Retrieved from https://www. ncbi.n1m.nih. gov/pubmed/28760549

Orua, S. (2013). Effects of COPD on cognitive functions: a case control study]. Tüberküloz Ve Toraks, 61(3), 193.

Roberto, D. N., Luca, B., Silvia, T., Fernanda, B., \& Paola, T. (2014). Extent and prevalence of cognitive dysfunction in chronic obstructive pulmonary disease, chronic non-obstructive bronchitis, and in asymptomatic smokers, compared to normal reference values. International Journal of Chronic Obstructive Pulmonary Disease, 9, 675-683.

Roncero, C., Campuzano, A. I., Quintano, J. A., Molina, J., \& Miravitlles, M. (2016). Cognitive status among patients with chronic obstructive pulmonary disease. International Journal of COPD, 11(1), 543.

Rubinov, M. , \& Sporns, 0. (2010). Complex network measures of brain connectivity: Uses and interpretations. NeuroImage, 52, 1059-1069.

Spilling, C. A., Bajaj, M. P. K., Burrage, D. R., Ruickbie, S., Thai, N. J., Baker, E. H. , . . . Dodd, J. W. (2019). Contributions of cardiovascular risk and smoking to chronic obstructive pulmonary disease (COPD)-related changes in brain structure and function. International Journal of Chronic Obstructive Pulmonary Disease, 14, 1855-1866. doi:10.2147/copd. S213607 
Tzourio-Mazoyer, N., L., B., Papathanassiou, D., Crivello, F., Etard, 0., Delcroix, N. . . . Joliot, M. (2002). Automated anatomical labeling of activations in SPM using a macroscopic anatomical parcellation of the MNI MRI singlesubject brain. NeuroImage, 15(1), 273-189.

Villeneuve, S., Pépin, V., Rahayel, S., Bertrand, J. A., \& Gagnon, J.-F. (2012). Mild cognitive impairment in moderate to severe COPD: a preliminary study. Chest, 142(6).

Wang, C., Ding, Y., Shen, B., Gao, D., An, J., Peng, K., . . . Qiu, S. (2017). Altered Gray Matter Volume in Stable Chronic Obstructive Pulmonary Disease with Subclinical Cognitive Impairment: an Exploratory Study. Neurotoxicity Research, 31(4), 453-463.

Ward, R. J., Lallemand, F., Witte, P. D., \& Dexter, D. T. (2008). Neurochemical pathways involved in the protective effects of nicotine and ethanol in preventing the development of Parkinson's disease: Potential targets for the development of new therapeutic agents. Progress in Neurobiology, 85(2), 135-147.

Wen, Z. , Marsh, H. W. , \& Hau, K. T. (2010). Structural Equation Models of Latent Interactions: An Appropriate Standardized Solution and Its Scale-Free Properties. Structural Equation Modeling A Multidisciplinary Journal, 17(1), $1-22$.

Yang, L. Y., Xiao, Z. W., Liu, X. R., Weng, X. C. , \& Zhang, X. X. (2006). Different roles of the left inferior prefrontal cortex in visual Chinese word recognition. Chinese Journal of Medical Imaging Technology.

Zhang H, Wang X, \& Lin J. (2012). Grey and white matter abnormalities in chronic obstructive pulmonary disease: a case-control study BM J Open, 2(2), e000844. 


\section{$532 \quad$ Figure legends}

533 Figure 1. The attention ability of the four groups (COPD-Smoking/COPD-

534 Nonsmoking/NonCOPD-Smoking/NonCOPD-NonSmoking) was compared (error bars:

535 SE). The $p$ values were adjusted by Bonferroni's correction for multiple comparisons.

$536 \quad(*: p<0.05 ; * *: p<0.01 ; * * *: p<0.001)$. 
537 Figure 2. The differences in the ReHo between the COPD and NonCOPD groups are 538 shown in axial, sagittal and coronal sections. Six clusters were found on whole brain T 539 test analysis, and the red areas indicate higher ReHo values. a) Left fusiform gyrus; $b$ )

540 Right fusiform gyrus; c) Left anterior cerebellum; d) Pons; e) Left inferior temporal 541 gyrus (cluster-1); f) Left inferior temporal gyrus (cluster-2). 
542 Figure 3. The ReHo of the left fusiform gyrus completely mediates the influence of

543 smoking on attention ability. The regression coefficient is shown in the figure. (*:

$544 \quad p<0.05 ; * *: p<0.01)$. 
Figures

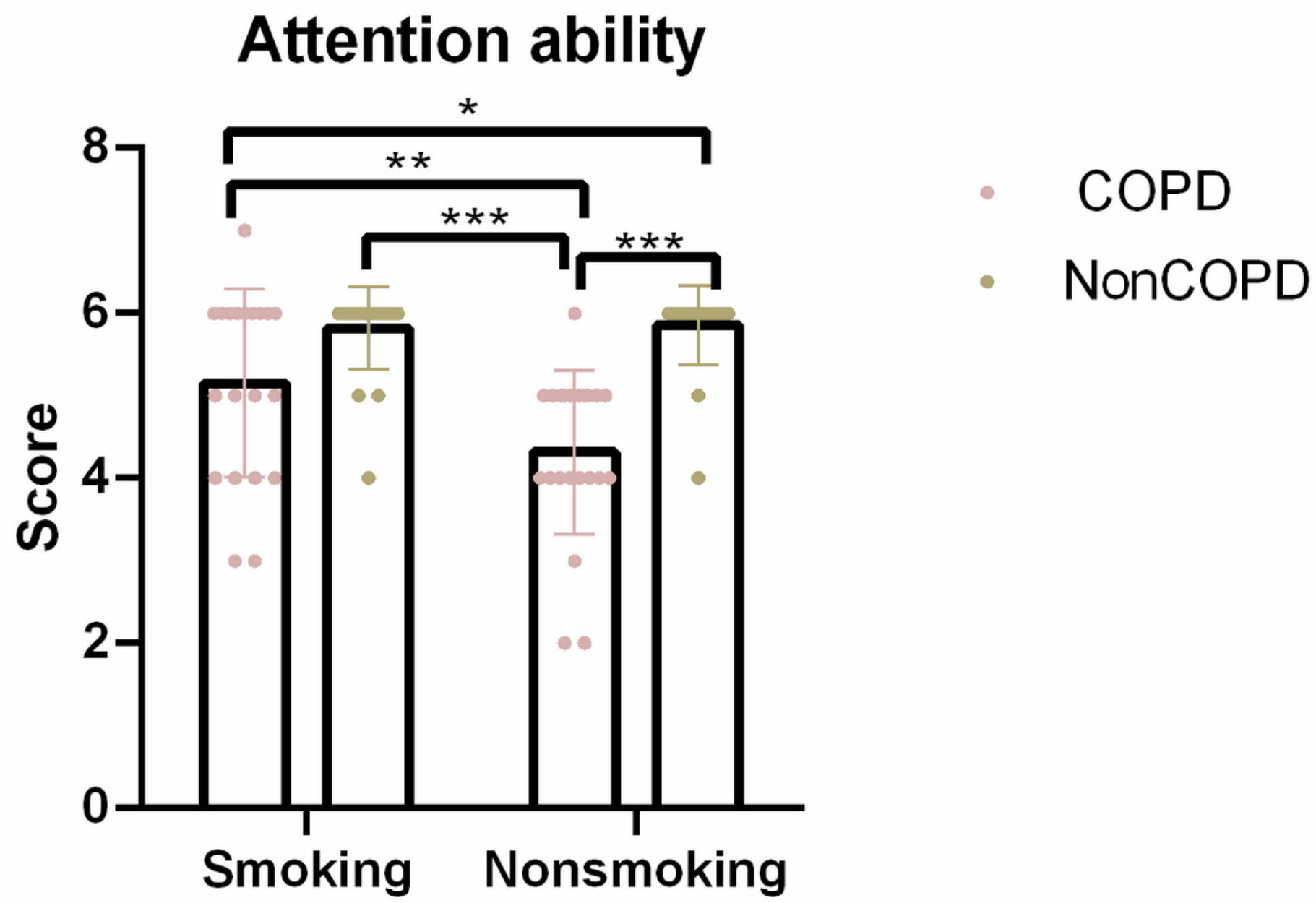

Figure 1

The attention ability of the four groups (COPD-Smoking/COPD-Nonsmoking/NonCOPD-

Smoking/NonCOPD-NonSmoking) was compared (error bars: SE). The $p$ values were adjusted by Bonferroni's correction for multiple comparisons. ( ${ }^{*} p<0.05$; $\star \star *: p<0.01$; $\left.* \star \star: p<0.001\right)$. 

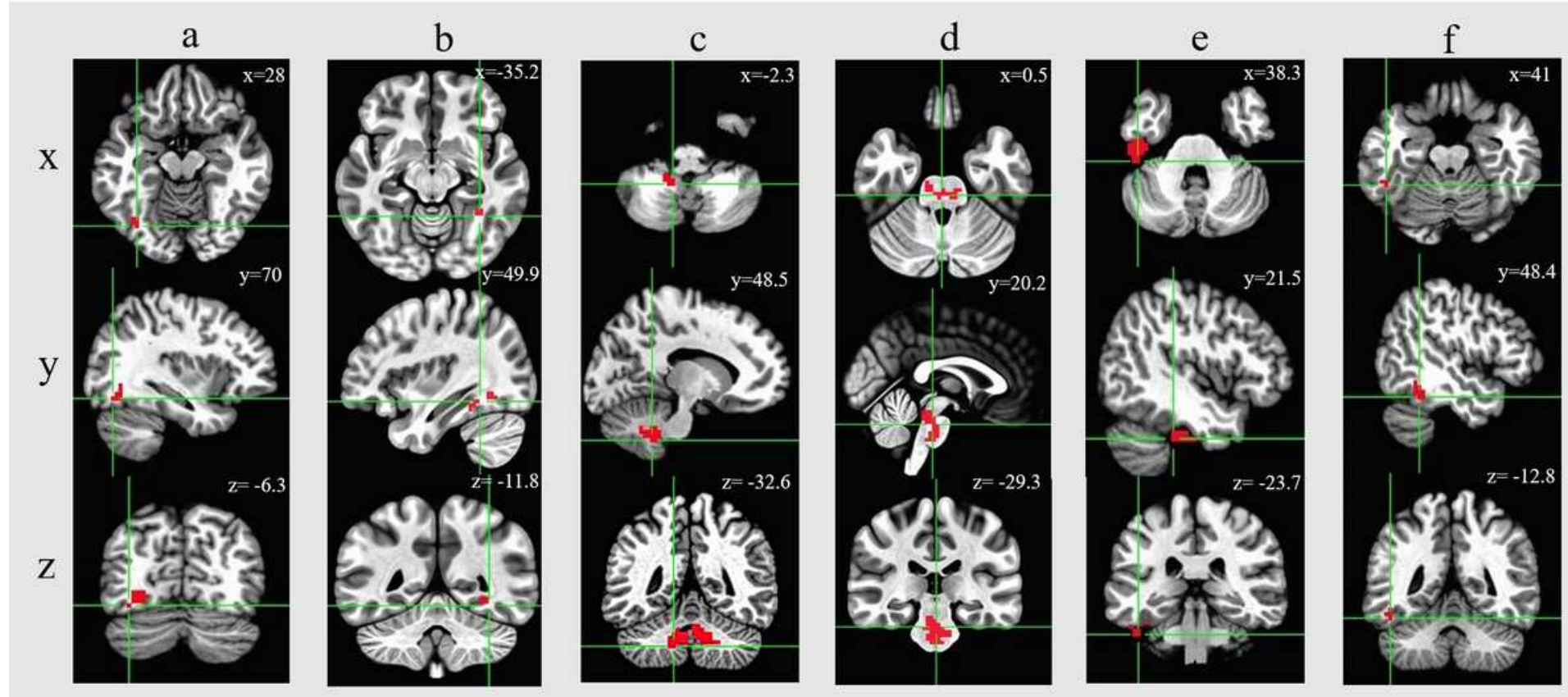

Figure 2

The differences in the ReHo between the COPD and NonCOPD groups are shown in axial, sagittal and coronal sections. Six clusters were found on whole brain $\mathrm{T}$ test analysis, and the red areas indicate higher ReHo values. a) Left fusiform gyrus; b) Right fusiform gyrus; c) Left anterior cerebellum; d) Pons; e) Left inferior temporal gyrus (cluster-1); f) Left inferior temporal gyrus (cluster-2).

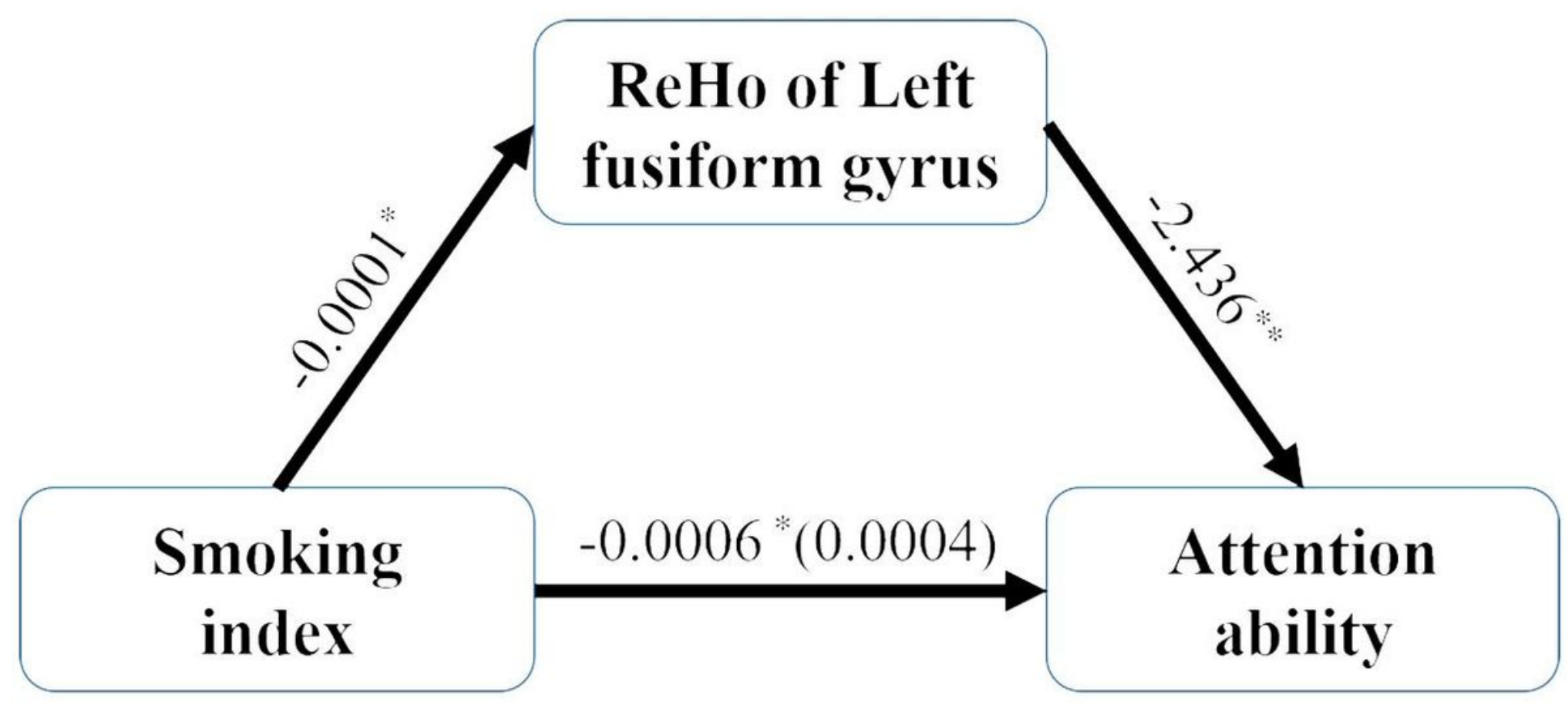




\section{Figure 3}

The ReHo of the left fusiform gyrus completely mediates the influence of smoking on attention ability. The regression coefficient is shown in the figure. $(*: p<0.05 ; * *: p<0.01)$.

\section{Supplementary Files}

This is a list of supplementary files associated with this preprint. Click to download.

- BIBChecklist0608.docx

- SUPPLEMENTARYMATERIAL0608.docx 